\title{
Experimental study of canard UAV aerodynamics
}

\author{
Hristian Panayotov ${ }^{1 *}$, Stanimir Penchev ${ }^{1}$, and Dancho Kolibarov ${ }^{1}$ \\ ${ }^{1}$ Technical University - Sofia, Plovdiv Branch, Department of Transport and Aviation Engineering, 4000 Plovdiv, Bulgaria
}

\begin{abstract}
The present paper presents the aerodynamic characteristics of a canard fixed-wing unmanned aircraft TERES-02. A wind tunnel experiment is conducted using a specially designed model of the aircraft. The model is produced through the methods of rapid prototyping using a FDM 3D printer. Aerodynamic corrections are made and thorough analysis and discussion of the results is carried out. The obtained results can be used to determine the accuracy of numerical methods for analysis of aircraft performance.
\end{abstract}

\section{Main properties of TERES-02 UAV}

TERES-02 (from TElesys REconnaissance and Surveillance) is a Reconnaissance and Surveillance unmanned aerial vehicle (UAV) produced by TELESYS Ltd - Plovdiv, Bulgaria and designed in collaboration with the Plovdiv Branch of TU-Sofia whose maiden flight was held successfully on July 2014 (Fig. 1).

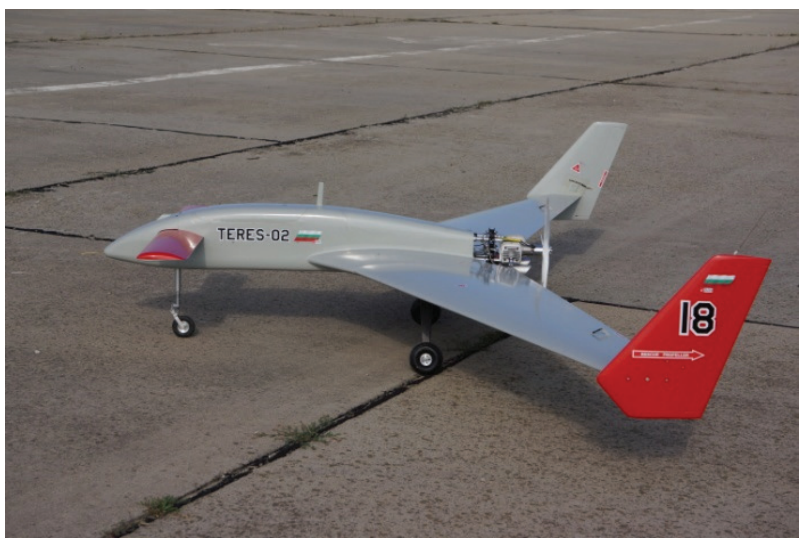

Fig. 1. TERES-02 UAV.

The aircraft itself features canard or tail-first aerodynamic arrangement with swept wing and tipmounted fins propelled by two-stroke, two-cylinder boxer engine with three-blade pusher propeller.

\subsection{Dimensions and performance}

The main dimensions and performance data are given in Table 1.

However, in order to fully analyse aircraft performance some basic aerodynamic characteristics have to be determined a priory. This could be done either theoretically (using numerical methods or analytical calculations) or experimentally. Even if theoretical calculations are used experimental validation of accuracy is also recommended. Hence an aerodynamic experiment is a must in order to properly determine aircraft performance.

Table 1. Dimensions and performance of TERES-02.

\begin{tabular}{|c|c|c|}
\hline Wingspan & 3840 & $\mathrm{~mm}$ \\
\hline MTOW & 55 & $\mathrm{~kg}$ \\
\hline Payload Weight & $>20$ & $\mathrm{~kg}$ \\
\hline Maximum Airspeed & 240 & $\mathrm{~km} / \mathrm{h}$ \\
\hline Cruising Speed & 110 & $\mathrm{~km} / \mathrm{h}$ \\
\hline Flight Endurance & $>8$ & $\mathrm{hours}$ \\
\hline
\end{tabular}

\subsection{Geometry of the wind tunnel model}

A 1:10 scale model of the real aircraft is designed to conduct an aerodynamic experiment. This is defined by the dimensions of the test section $(600 \times 400 \mathrm{~mm})$ of the wind tunnel (Fig. 6). The maximum allowed wingspan of the model is $400 \mathrm{~mm}$ and maximal cross-sectional area is $1800 \mathrm{~mm}^{2}$. Also a special support of the model is designed to ensure attachment of the model itself to the internal aerodynamic balances. The main requirements towards the support are: minimum parasite drag; minimum interference with the model; minimum crosssectional area; maximum strength and stiffness.

A semi-view of the 3D model of the assembly model and support is shown on Fig. 2. The propeller and the lending gear are not modelled. Fins are modelled as a separate part from the aircraft and will be produced alone. The support is attached to the model with a screw mounted at the top of the fuselage. The support has mountable nose to ensure access to internal aerodynamic balance attachment screw.

\footnotetext{
${ }^{*}$ Corresponding author: hristian@tu-plovdiv.bg
} 


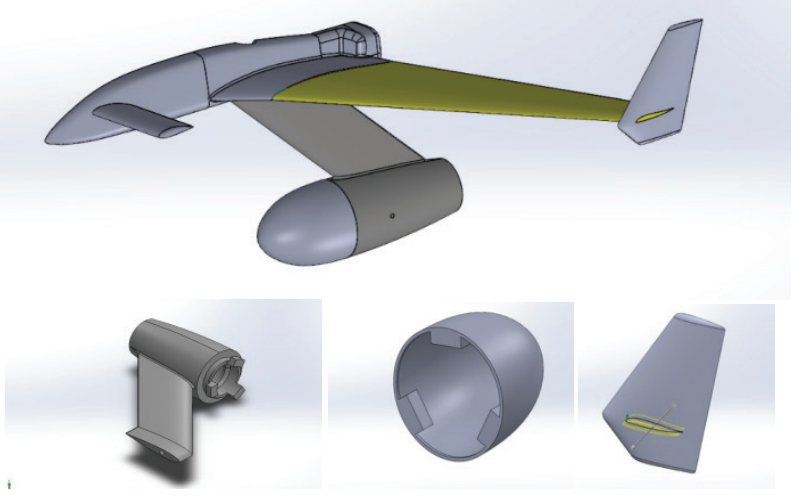

Fig. 2. Experimental wind-tunnel 3D model and its parts.

\section{Production of the model}

The designed wind tunnel model (Fig. 2) is to be produced using FDM (Fused Deposition Modeling or Fused Filament Fabrication) 3D printer followed by finish work. The chosen material is ColorFabb XT-CF20 carbon fiber reinforced polymer that possesses high strength and stiffness. Also the 3D printed parts surface is rough or "shagreen" so it is appropriate to be finished with filler spray and finally polished. Wanhao Duplicator $5 \mathrm{~S}$ is the $3 \mathrm{D}$ printer used. It is a high precision, high resolution machine with enough build volume dimensions to create wind tunnel models.

Before printing the model it has to be "sliced" by software to produce numerical control code for the printer. Fig. 3 shows the sliced model of the fuselage and wing part. Here the operation regimes of the printer are controlled as well. The nozzle diameter is $0,4 \mathrm{~mm}$, the layer resolution is $0,1 \mathrm{~mm}$, the $\mathrm{X}, \mathrm{Y}$ precision of head positioning is 12,5 microns. The temperature of the nozzle is $240^{\circ} \mathrm{C}$ and the temperature of the heated bed is $75^{\circ} \mathrm{C}$ to avoid temperature stress in the material and following deformations during fabrication.

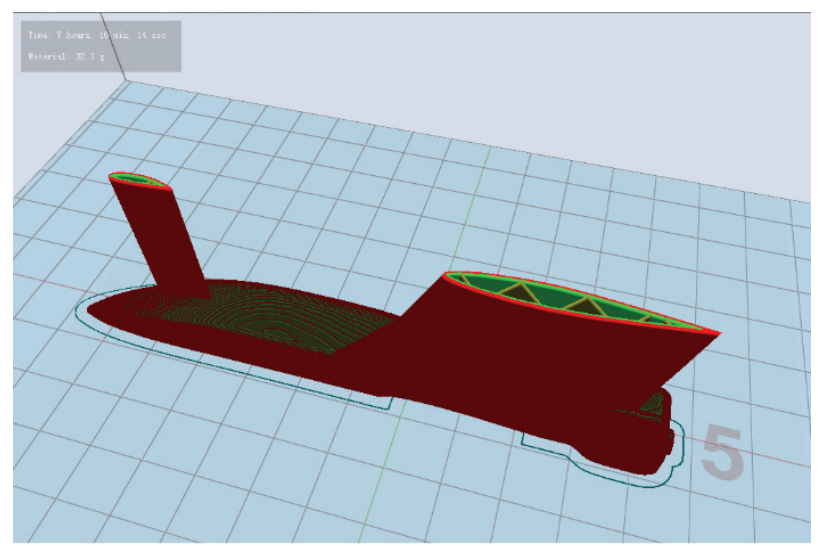

Fig. 3. 3D printer slicer software.

Fig. 4 shows the "raw" printed parts for the model without the finish work and Fig. 5 shows the finished model in the test area of the wind tunnel. The model is mounted onto internal aerodynamic balances.

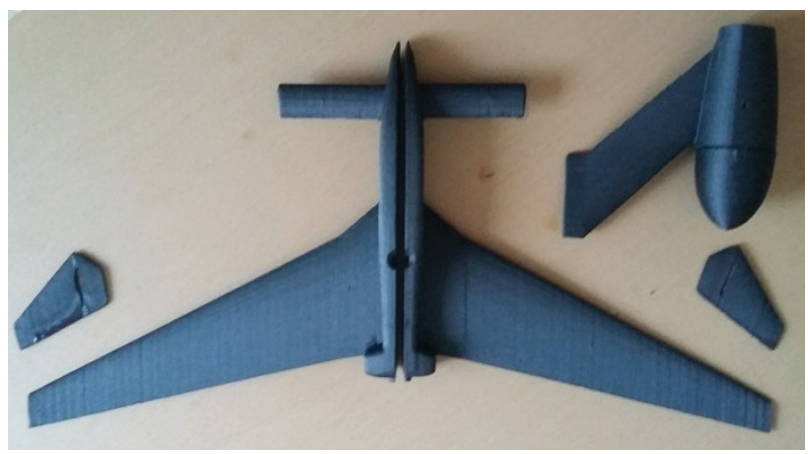

Fig. 4. Printed parts for the wind tunnel model.

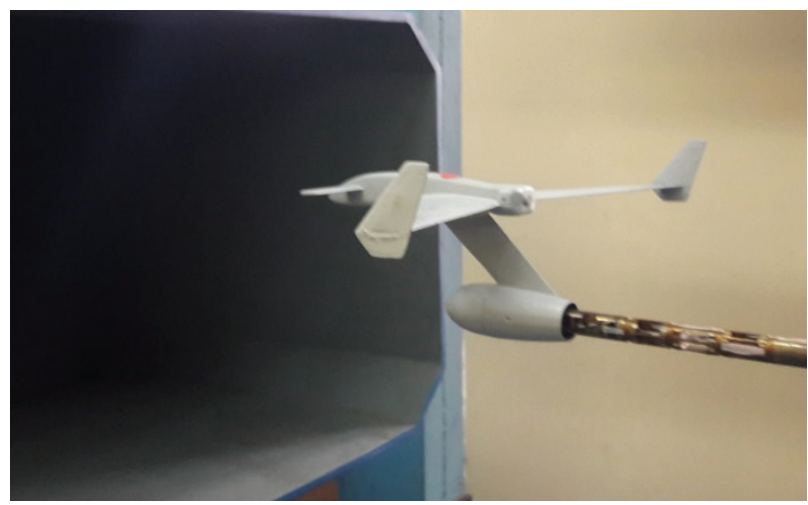

Fig. 5. Finished model in the test area.

\section{Methodology}

\subsection{Wind tunnel}

The ULAK-1 wind tunnel located at the Laboratory of Aerodynamics in the Plovdiv Branch of TU-Sofia has a low Reynolds number, an open test area, a closed-loop research facility (Fig. 6).

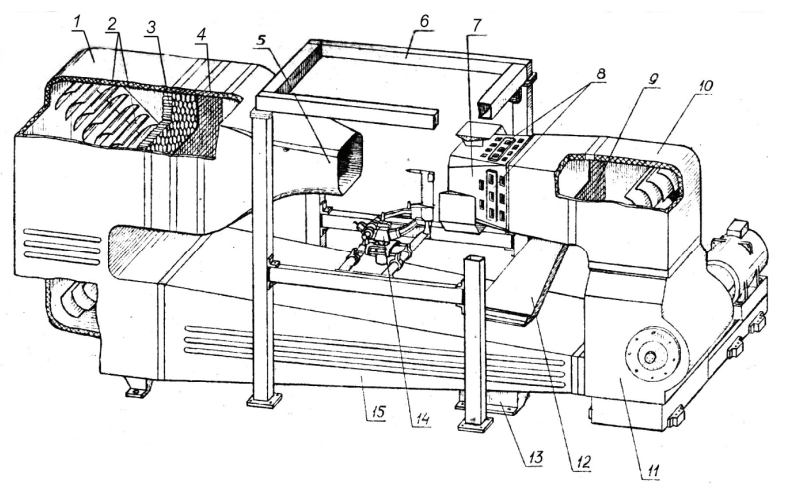

Fig. 6. ULAK-1 wind tunnel.

The maximum airflow speed in the test area is 50 $\mathrm{m} / \mathrm{s}$, the dimensions of the cross section of test area are $600 \times 400 \mathrm{~mm}$. Aerodynamic forces and moments are measured by internal six-component tensiometric aerodynamic balances. The angle of attack and sideslip angle are controlled by $\alpha-\beta$ mechanism. The experiment control and measurements are fully automated and computerised. 


\subsection{Measured properties}

In the present study the aerodynamic characteristics in the plane of symmetry are analysed in terms of the angle of attack $-\alpha$ at a given Reynolds number $-R e$ :

$$
\left\{L, D, M, c_{L}, c_{D}, c_{M}\right\}^{T}=f(\alpha, R e),
$$

where: $L$ is lift force; $D$ is drag force; $M$ is pitch moment and $c_{L}, c_{D}, c_{M}$ respectfully are their aerodynamic coefficients.

Aerodynamic balances measure directly the forces and moments in the coordinate system of the balances: axial force $X$, normal force $Z$ and pitch moment $M_{y}$. The aerodynamic coefficients in the coordinate system of the balance are calculated using the following corelations [1]:

$$
c_{x}=\frac{X}{q S} ; c_{z}=\frac{Z}{q S} ; c_{m_{y}}=\frac{M_{y}}{q S c},
$$

where $S$ is the reference area; $c$ is reference length (mean aerodynamic chord) and $q$ is dynamic pressure $(q=$ $\left.\frac{\rho V_{\infty}^{2}}{2}\right)$, where $V_{\infty}$ is free stream velocity and $\rho$ is density of the air.

Once the coefficients (2) are measured the aerodynamic coefficients in the wind coordinate system can be calculated using the transformations:

$$
\begin{gathered}
c_{D}=c_{x} \cos \alpha+c_{z} \sin \alpha ; \\
c_{L}=c_{z} \cos \alpha-c_{x} \sin \alpha ; \\
c_{m}=c_{m_{y}}+\frac{\left(z_{0}-z_{G}\right) c_{x}}{c}-\frac{\left(x_{0}-x_{G}\right) c_{z}}{c},
\end{gathered}
$$

where index " 0 " and " $G$ " indicate the location of the coordinates of the balances moment centre and the model respectfully.

\subsection{Aerodynamic corrections}

The "raw" results from the aerodynamic experiment have to be corrected to eliminate various sources of errors [2]. The most significant of them for the ULAK-1 wind tunnel are corrections of the turbulence factor (TF) and correction from the downwash of the flow in the test area.

The first correction defines the ratio between the intensity of the turbulence in the test area and the free atmosphere turbulence [2]. Previously conducted experiments in ULAK-1 show that the turbulence factor is $T F=2,14$ [3]. Hence the measured Reynolds number has to be corrected so that it yields the effective Reynolds number

$$
R e_{e f f}=T F \cdot R e .
$$

The second correction eliminates the influence of the downwash in the test area flow. Different methods are used to define the downwash angle in the test area. In the present study the model is tested in two different configurations at various angles of attack - normal and inverted (the model is positioned upside down) flight.
Provided the downwash angle in the test area is zero the results will be the same. Otherwise, the asymmetry will cause different results for each angle of attack and thus the zero-lift angle of attack will be different in normal and inverted flight. Finally, the downwash angle in the test area will be

$$
\varepsilon_{T A}=\frac{\alpha_{0_{\text {nor }}}-\alpha_{0_{\text {inv }}}}{2}
$$

and the true angle of attack in the test area is

$$
\alpha_{\text {true }}=\alpha-\varepsilon_{T A} .
$$

The measured aerodynamic coefficients then have to be corrected:

$$
\begin{gathered}
c_{D_{\text {true }}}=c_{D} \cos \varepsilon_{T A}+c_{L} \sin \varepsilon_{T A} ; \\
c_{L_{\text {true }}}=c_{L} \cos \varepsilon_{T A}-c_{D} \sin \varepsilon_{T A} .
\end{gathered}
$$

\section{Results and discussion}

Three separate experiments were conducted following the above-mentioned methodology: normal flight and inverted flight to determine the downwash angle in the test area and drag polar measurement of the support. The drag of the support is taken in account and is subtracted from the total drag of the model cum support. Lift and drag coefficients of the support are given in Table 2. The interference drag between the aircraft and the support is neglected.

For all experiments the reference area of the model and the reference length are: $S=0,018 \mathrm{~m}^{2}$; $c=0,053 \mathrm{~m}$. The air density is $\rho=1,1652 \mathrm{~kg} / \mathrm{m}^{3}$. The effective Reynolds number based on the mean aerodynamic chord $c$ calculated using (4) is $R e_{\text {eff }}=190000$.

Table 2. Aerodynamic characteristics of the support

\begin{tabular}{|c|c|c|c|c|c|}
\hline$\alpha, \operatorname{deg}$ & $c_{D}$ & $c_{L}$ & $\alpha, \operatorname{deg}$ & $c_{D}$ & $c_{L}$ \\
\hline$-7,91$ & 0,0072 & $-0,0049$ & 6,07 & 0,0067 & 0,0052 \\
\hline$-5,91$ & 0,0070 & $-0,0051$ & 8,07 & 0,0082 & 0,0105 \\
\hline$-3,92$ & 0,0068 & $-0,0054$ & 10,06 & 0,0110 & 0,0158 \\
\hline$-1,91$ & 0,0065 & $-0,0055$ & 12,06 & 0,0102 & 0,0210 \\
\hline 0,08 & 0,0061 & 0,0059 & 14,05 & 0,0126 & 0,0207 \\
\hline 2,08 & 0,0079 & 0,0056 & 16,04 & 0,0147 & 0,0258 \\
\hline 4,08 & 0,0065 & 0,0054 & 18,04 & 0,0156 & 0,0254 \\
\hline
\end{tabular}

Calculations based on formula (5) yield downwash angle in the test area $\varepsilon_{T A}=1,2$ degrees, which is consistent with similar previous measurements [4]. After 
corrections (5) and (6) the final results for aerodynamic characteristics are shown graphically in Figures 7 to 10 .

Fig. 7 depicts the variation of the lift and drag coefficients of the aircraft in terms of the angle of attack. It can be seen that the lift coefficient curve does not reach its maximum value and the stall angle of attack is not achieved. This is due to the limited maximal angle of attack for ULAK-1 (18 deg) and the downwash angle additionally reduces it. Also the swept wing needs higher angle of attack to stall, so a new support has to be designed to allow higher angles of attack. The minimum drag coefficient is $c_{D \min }=0,0407$, which is quite high for a "clean" configuration of aircraft but this can be explained with the low Reynolds number.

\section{$\operatorname{Re}=190000$}

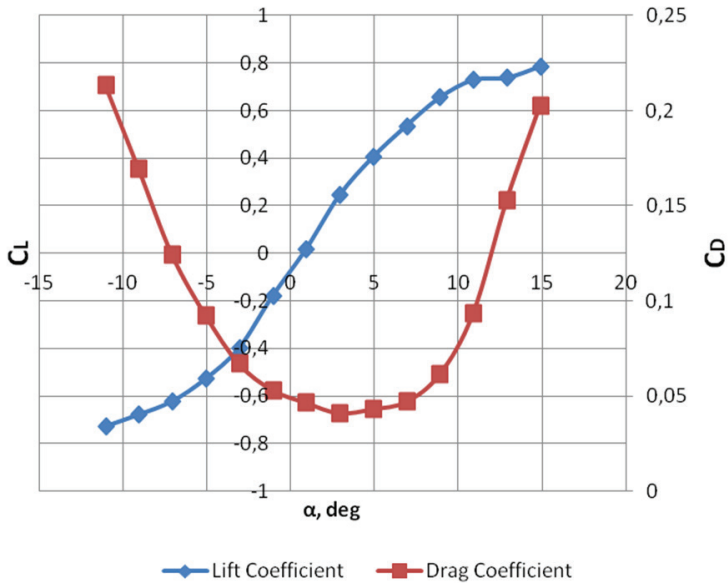

Fig. 7. Lift and Drag Coefficients vs. Angle of Attack.

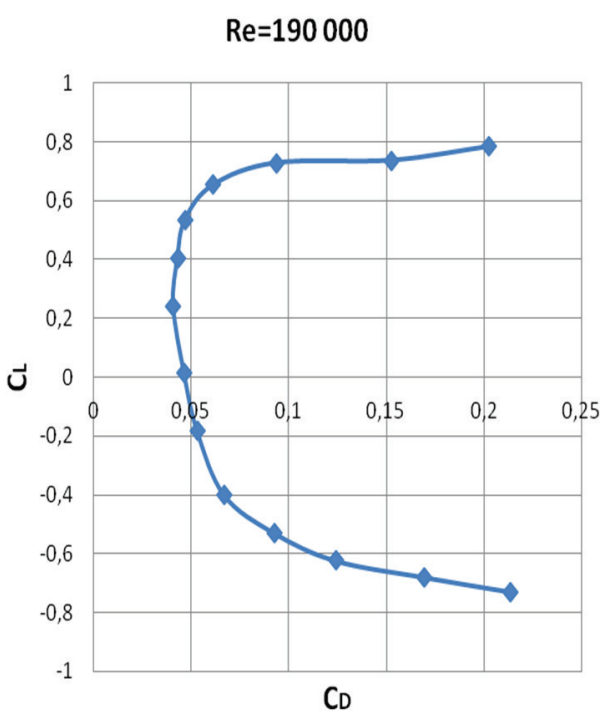

Fig. 8. Drag Polar

The relatively high drag coefficient influences the values of the Lift-to-Drag ratio (Fig. 9). The maximum value is close to 12 at $7 \mathrm{deg}$. angle of attack. The pitch moment coefficient (Fig. 10) is calculated about the aircraft centre of gravity and shows typical pattern for a statically stable aircraft.

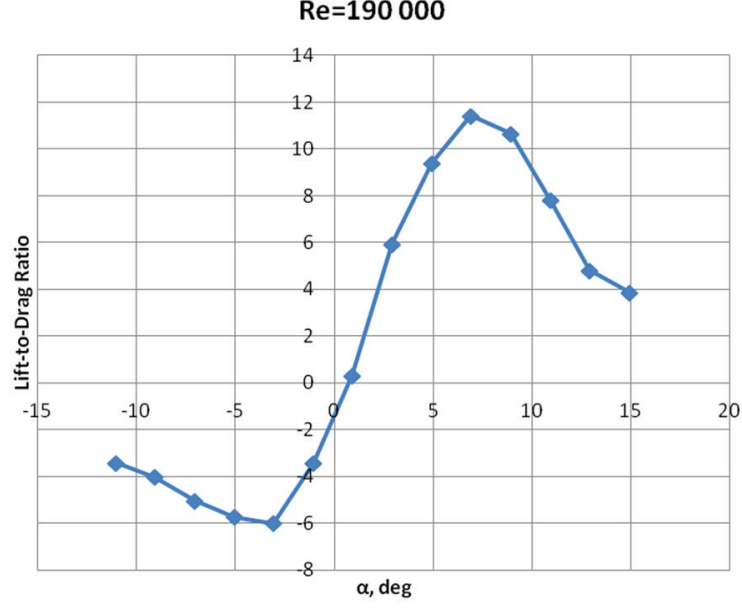

Fig. 9. Lift-to-Drag Ratio vs. Angle of Attack.

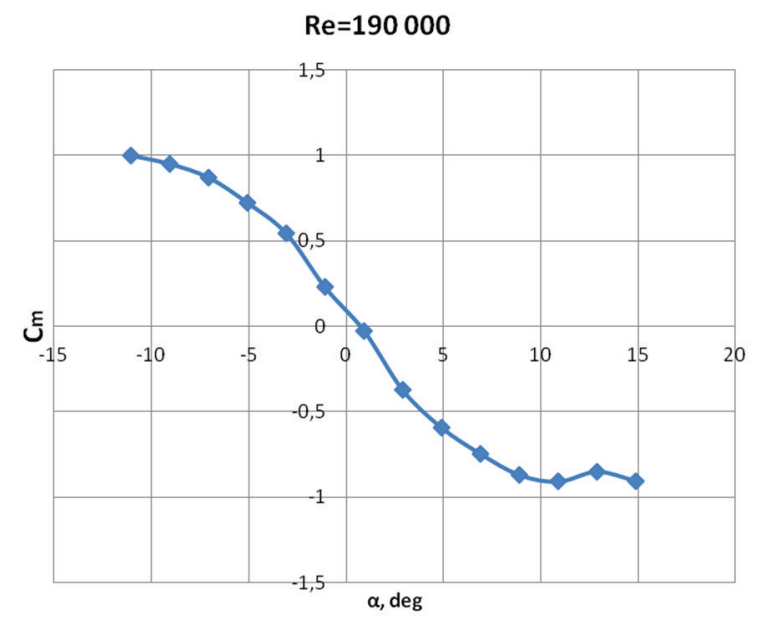

Fig. 10. Pitch Moment Coefficient vs. Angle of Attack.

\section{Conclusion}

Aerodynamic experiments were held with a specially designed model of TERES-02 UAV. The results will be used for calculation of aircraft performance and validation of numerical simulations.

\section{References}

1. J. Anderson Jr., Fundamentals of aerodynamics, (McGraw-Hill, 2001)

2. J. Barlow, W. Rae, A. Pope, Low-Speed Wind Tunne Testingl, (John Wiley \& Sons, 1999)

3. S. Penchev, D. Seyzinski, D. Kolibarov, Flow parameters investigation in the test area of wind tunnel ULAK-1 (UT-1), Mechanics of Machines 62 9-12 (2006)

4. D. Zafirov, H. Panayotov, Joined-wing test bed UAV, CEAS Aero J. 6 137-147 (2015) 